\title{
Identification of factors associated with various types of impaired driving
}

Mahdi Rezapour $^{1 凶} \&$ Khaled Ksaibati ${ }^{2}$

Despite significant progress in reducing impaired driving, impaired drivers are still a public threat to themselves and others. Studying all types of drivers' impairment is especially important as impairment in the literature has been mainly looked at from an angle of the driving under the influence (DUI). In other words, there is a definitional ambiguity regarding the impaired driving in the literature review. Addressing that unclarity is especially important as while many considered impaired driving such as distraction and fatigued driving are similar to DUI, they account for a higher proportion of fatalities on roadway, compared with DUI. Thus, first we highlight links between DUI and other impaired driving based on the literature review, and then this study highlighted factors associated with various impaired driving so drivers' actions and behaviors could be addressed more efficiently. Here, in addition to DUI, impaired drivers are defined as whether drivers were fatigued, distracted, or under some emotional conditions at the time of crashes. Multinomial logit model (MNL) was extended to a model excluding or equating some coefficients across categories, making parameters' estimates more robust. The analysis results highlighted, for instance, types of vehicles that the drivers own, drivers' gender, age, seatbelt use, and various drivers' actions such as failure to keep the proper lane and following too close are associated with various impaired drivers' actions. These associations are expected to be related to unseen drivers' characteristics that were not recorded the time of crashes. This is one of the earliest comprehensive studies, investigating the association of various factors and different types of impaired driving.

\footnotetext{
${ }^{1}$ Independent Researcher, Mashhad, Iran. ${ }^{2}$ University of Wyoming, 1000 E. University Avenue Department 3295, Laramie, WY 82071, USA.

凶email: rezapour2088@yahoo.com
} 


\section{Introduction}

/ ehicle crashes are one of the leading causes of death worldwide, where more than 1 million people die and 20 million more people are severely injured (World Health Organization, 2007). In addition, crashes are ranked 7th in terms of life lost (Subramanian, 2005), equivalent to 230 billion dollars' worth of crash costs every year. Among all factors contributing to crashes, human factors play a crucial role, beyond all other factors, such as environmental and vehicle characteristics (Safety, 2003). For example, about $30 \%$ of all U.S. motor vehicle fatalities are due to the human factor of alcohol-impaired drivers alone (National Highway Traffic Safety Administration, 2017); also, the contribution of fatigued and distracted drivers $(37 \%)$ to traffic fatalities has been higher than driving under the influence (DUI) (30\%).

It is not only the presence of alcohol or drugs in the blood that impairs the performance of drivers but also factors such as distraction, fatigue, or emotional conditions of drivers. While a significant emphasis has been given to DUI, there are definitional ambiguity for the impaired driving. Here DUI is defined as driving under the influence of drug or alcohol.

Past studies defined impairment not necessarily from DUI point of view. For instance, impaired drivers include drivers compromised by a disabling physical or cognitive condition (Berger et al., 2000). In another study, patients with medical conditions such as epilepsy and diabetes that create a risk of losing control while driving were defined as impaired (Aschkenasy et al., 2006).

However, drowsiness or fatigue driving, for instance, lead to severe vehicular crashes. For instance, sleepiness or drowsiness affects drivers' functioning in crashes because drivers are less attentive, have slower reactions, and have impaired judgment (Lyznicki et al., 1998).

Study objectives. The first objective of this study is to highlight that there are other impaired driving behaviors that are as risky as DUI, and thus should be given more attention. This objective is achieved by conducting a literature review to highlight the similarity between those impaired driving behaviors and DUI. Studying all impaired driving are important as they account for almost half of the fatalities in the state of Wyoming during the study period, while those impaired driving account for $10 \%$ of all crashes.

The second objective is to investigate the similarities and dissimilarities between associated factors to DUI and other impaired driving actions. To achieve that objective, we disaggregated impaired drivers, instead of considering the aggregated version, and conducted a unified model of multinomial model. That is especially important as it has been highlighted that factors influencing one risky driving behavior do not necessarily influence other risky behaviors (Fernandes et al., 2010); where in that study, different predictors for speeding, drink-driving and fatigued driving were highlighted.

The third objective is including the predictors to foresee or suggest driver impairment. To achieve that objective, we indirectly incorporate other unseen factors that seem likely to be associated with drivers' attributes. For instance, vehicle types, environmental characteristics, vehicle types could provide information regarding the characteristics of drivers.

Finally, it should be noted that this study was conducted to investigate the association, but not necessarily the causations, where different forms of impaired driving including drug/alcohol, fatigued, distraction, and emotional drivers were incorporated. The study was conducted in the western state of the US, which is characterized by mountainous geometric characteristics. This is one of the earliest studies that challenge the inconclusive definition of impaired driving, by comprehensively examining the association between various factors and including various types of impaired drivers.

\section{Literature review}

As in this study, a few categories related to impaired drivers were considered, where previous studies considered those factors individually; the literature review will be presented in the subsections related to response categories, including DUI, fatigue, distraction, and emotional conditions of drivers.

Driving under the influence (DUI). It is essential to include alcohol-related crashes as they total more than 40 billion dollars in crash-related costs annually (Blincoe et al., 2015). A study by means of systematic review of studies assessing the risk of crashes due to other drugs such as cocaine and cannabis was conducted (Elvik, 2013). The results showed a significant effect of drugs on severe and fatal crashes compared with less critical crashes.

The relationship between alcohol and driver characteristics in motor vehicles crashes was investigated (Abdel-Aty and Abdelwahab, 2000). The factors considered in that study were age, gender, race and residency of drivers. The results showed that the 24-34 age group experiences the highest alcohol/drug involvement rate in crashes. In addition, male, white, and instate drivers are more involved with alcohol and drugs. However, no attempt was made to fit the included log-model with more than three variables.

The intersection of DUI, criminal history, and psychiatric comorbidity was investigated in another study (Nelson et al., 2019). Results of computerized mental health assessment and criminal record during the last $4-5$ years were found to be significant risk factors for arrest among DUI offenders.

Although extensive efforts have been made to understand the association between different drugs or alcohol use and the severity of crashes, there is a limited investigation of the associated factors of DUI itself.

Fatigued drivers. Consideration and evaluation of fatigued driving are essential as fatigued driving is common, especially in the U.S. For instance, about half of the U.S. adult drivers admitted that they often feel drowsy while getting behind the wheels, while $20 \%$ admit they fall asleep behind the wheels (The National Sleep Foundation, 2021).

The similarity between fatigued drivers and DUI drivers can be easily made. In one study, the effects of fatigue compared to alcohol consumption on train engineers' performance were evaluated (Lowrie and Brownlow, 2020). It was found that the impairment due to fatigue was in a close range to the impairment of moderate alcohol consumption. Another study evaluated the similarity of alcohol intoxication and sleepiness on driving (Lowrie and Brownlow, 2020). The results found that the mean reaction time of sleep deprivation is slower compared with alcohol of $50 \mathrm{mg}$ of alcohol per $100 \mathrm{ml}$ of blood.

The importance of drowsiness on the risk of motor vehicle crashes was investigated and considered a type of impaired driver (Cummings et al., 2001). The results showed that fatigue resulted in participants disengaging and operating the simulator unsafely. In addition, in that study, impairment due to fatigue was similar to the impairment associated with a moderate level of alcohol consumption.

Driver risk factors for sleep-related crashes were evaluated in another study (Stutts et al., 2003). The data was collected using brief telephone interviews for those drivers that recently involved 
in sleep-related crashes. The results showed that drivers in sleeprelated crashes are more likely to work multiple jobs or working night shifts.

Effects of fatigued driving and drunk driving on drivers' physical characteristics was considered in another study (Zhang et al., 2014). The results highlighted that both fatigue and drunk driving impair drivers' physical characteristics. Despite the importance of drowsy driving, few efforts have been made to emphasize it as a factor contributing to a risky driver's condition. Fatigued driving is considerable and deserves more investigation to highlight its associated factors.

Distraction. The similarity between distracted drivers and DUI was made in a past study (Lerner, 2011), where it was discussed that the distraction can pose as much risk as the DUI drivers. Driver distractions have been defined as diverting attention away from activities essential for safe driving toward other activities (Regan and Mitsopoulos, 2001). Drivers' distraction is not necessarily related to technologies inside vehicles, but it could also be related to events outside the vehicles, like being distracted by an animal or some sceneries on the roadside.

The injury severity of inattentive drivers compared with normal drivers was investigated in the past study (Zhao and Khattak, 2018). It was found that in single vehicle crashes the severity of crashes increase by $9.7 \%$, while in two-vehicle crashes, the probability of injury crashes increases by $14.6 \%$, compared with normal drivers. In another study, the most commonly reported distraction include: lack of concentration, adjusting invehicle equipment, looking at objects outside the vehicle, and talking to the other vehicles' occupants (McEvoy et al., 2006). Inattentive drivers also fail to pay proper attention to the roadway and hazard ahead while driving.

Limited studies were conducted to study associations of other factors and distraction. Driver distraction by digital billboards based on naturalistic sieving study (NDS) data was analyzed by the structural equation modeling (SEM) (Sheykhfard and Haghighi, 2020). Factors such as younger drivers, night, and unclear whether, like a rainy day, were some of the factors determined to increase the likelihood of drivers' distraction.

Also, extensive works have been done on mobile phone distracted drivers, where the attention of drivers were diverted by means of cell phone. For instance, the impact of cell phone position on driving and gaze behavior was investigated (Turnbull et al., 2021). The results highlighted that for scenarios that mobile phone is presented, participants shifted the fixation more frequently. The important impact of cell-phone use on drivers' behavioral adaptation was investigated (Oviedo-Trespalacios et al., 2017). The results highlighted that drivers took different speed adaptation based on different phone conditions, e.g., hands-free and handheld.

Emotional condition. This category accounted for various variables mainly related to emotional conditions such as anger, sadness, or depression. Those characteristics were recorded as an emotional condition at the time of crashes by the highway patrol. Minimal studies investigated that aspect of drivers while modeling crashes. The "cognitive theory of emotion" states that positive or negative attributes of emotions are based on the appraisals regarding the events that individuals encounter (Arnold, 1968). So, at the time of crashes, drivers, based on their personalities and attitudes, will evaluate the crash condition and take on emotion, which is recorded by the highway patrol.

The impact of various physical and emotional factors on car drivers' driving styles was investigated by survey design (Eboli et al., 2017). Using frequency distribution and correlation analysis, the authors concluded that the self-definition of driving style could not be reliable.

Anger is associated with more aggressive and risky driving behaviors in daily driving, more violations, and greater use of hostile and aggressive ways of expressing anger (Deffenbacher et al., 2003). In addition, it has been highlighted that mood states impact drivers' risky driving behavior through risk perception and, consequently, risk attitude (Hu et al., 2013).

Drivers' health and its impact on the crash involvement was investigated (Sagberg, 2006). The estimations were based on drivers with and without the conditions. The study found significant risk factors for crash involvement, including feeling depressed and taking medications.

Emotions have been discussed to impact an individual's risk perception, driving risk attitudes, and risky driving behaviors; in other words, while positive emotions reduce risky driving behaviors, the negative emotions increase them ( $\mathrm{Hu}$ et al., 2013). Furthermore, those negative feelings are expected to be influenced by various drivers' demographic characteristics and other attributes of crashes, highlighting the indirect impact of unseen factors. Also, the relationship between situational emotions such as anger and driving choice, and abilities was investigated (Abdu et al., 2012), highlighted that drivers have lack of control over incidents. Although not many study conducted to investigate the similarities between DUI and driver's emotion conditions, the similarities could be observed based on the above discussion that drivers under emotional conditions like DUI, are having impaired driving behaviors.

In summary, past studies have attempted to highlight factors of impaired driving while modeling the severity of crashes. However, the questions of what factors are associated with impaired driving are yet to be answered. Furthermore, despite the efforts in studying the impacts of various types of impaired driving on the severity or frequency of crashes, less attention has been made to understand the association of other factors to those impaired driving. Thus, this study was conducted to expand the understanding regarding factors to various impaired drivers in a unified modeling framework.

\section{Methods}

The method is presented in two main subsections. The first subsection will discuss the statistical method, and the second part will outline the framework and idea behind the implemented method.

Statistical analysis. Here, the multinomial logit model (MNL) was used due to the categorical nature of the response. Compared with the common method of the MNL proposed by (McFadden, 1973), which uses the maximum likelihood function for solving the algorithm, in the vectorized generalized linear model (VGAM), the model will be solved by means of the iteratively reweighted least square (IRLS), or maximization through the minimization of deviance. VGLM, similar to the generalized linear model $(\mathrm{GLM})$, the $\mathrm{j}$ th linear predictor of $\eta_{i}=g_{j}\left(\theta_{j}\right)$ is written as:

$$
\eta_{j}=\eta_{j}(x)=\sum_{p=1}^{P} \beta_{j p} x_{p}
$$

Where $p$ is a number of parameters to be estimated, and $j$ is number of the response category. The above based on the combination of all explanatory variables in a single matrix, and for iteration $a$, could be written as: $\eta^{(a)}=X_{\mathrm{VLM}} \beta a$. 
So, it is (Yee, 2010):

$$
\left(\begin{array}{c}
\eta_{1}\left(x_{i}\right) \\
\vdots \\
\eta_{j}\left(x_{i}\right)
\end{array}\right)=\left(\begin{array}{ccc}
\beta_{(1) 1} & \cdots & \beta_{(1) p} \\
\vdots & \ddots & \vdots \\
\beta_{(j) 1} & \cdots & \beta_{(J-1) p}
\end{array}\right) x_{i}
$$

The model parameters estimations based on the IRLS include creation of matrices of transformed response as $z^{(n)}=\eta_{i}^{(n)}+\left(W_{i}\right)^{(n)^{-1}} u_{i}^{(n)}$, Where $\left(u_{i}\right)_{j}=\frac{\partial \ell_{i}}{\partial \eta_{j}}$ is the score vector for $\mathrm{j}$ th element, and $\left(W_{i}\right)_{j k}=\frac{-\partial^{2} \ell_{i}}{\partial \eta_{j} \partial \eta_{k}}$. $\left(W_{i}\right)_{j k}$ measures the amount of information each observation carries. Then, the transformed or working response could be written as:

$$
z_{n-1}=\beta_{n} X_{\mathrm{VLM}}+\varepsilon_{n-1}
$$

For iteration $n, X_{\mathrm{VLM}}$ is formed using the Kronecker process to modify the $X$ matrices based on various matrices of constraints $H_{k}$, which is regressed based on a working response of $\left(z_{1}^{\top}, \ldots, z_{n}^{\top}\right)^{\top}$.

The generalized least square (GLS) system of equations is converted to the ordinary least square (OLS) by pre-multiplying both sides of the equation by the Cholesky decomposition, which standardizes the error terms and removes the correlations across them. The Cholesky decomposition is used to obtain the $U$ matrix, which is the square root of the weight, where the weight could be written as $W=U^{\mathrm{T}} U$. Now, the matrix of $U$ will be used for obtaining the OLS by multiplication of the left- and righthand sides of Eq. 3 by $U$. So, Eq. 3 turns to:

$$
z_{a-1}^{* *}=X_{\mathrm{VLM}_{a-1}}^{* *} \beta_{a}+\varepsilon_{a-1}^{* *}
$$

Now the above equation can be solved by the OLS.

The important part of the model is the application of matrices for setting the constraints. For instance, application of values of zero to remove the estimate of a parameter, using matrices of values of 1 for its column to constrain them to be equal, or using a diagonal symmetric matrix of $j \times j$ to estimate all parameters. In contrast, this has been done in most packages by relying on the maximum likelihood function by just constraining the parameters at their starting values.

The IRLS employs the Newton-Raphson method with Fischer scoring for an iterative solution to the likelihood function (Green, 1984). The model parameter estimates could be summarized as follows: first, preparation of the $X_{\mathrm{VLM}}$ from $X_{\mathrm{LM}}$, and various constraints matrices. Consequently, solving Eq. 4.

It should be noted that for the MNL, the application of Fischer scoring, or weight is equivalent to the Newton-Raphson method, where deviance is set as a criterion for minimization. The iteration continues until the changes in the objective function of deviance are smaller than the threshold value. Throughout the algorithm, $\theta$ in $\eta_{i}=g_{j}\left(\theta_{j}\right)$ and $\eta$ will be switched by using the inverse of the equation.

Again, after transforming both sides of the model, $X_{\mathrm{VLM}}$ and $z$ in Eq. 4 , the $z$ will be regressed based on the transformed version of $X_{\mathrm{VLM}}$ based on the OLS, and $\eta$ would be the fitted values (Yee, 2010). Finally, the sum of the log of the probability mass function (PMF) of the multinomial distribution will be used for estimating the log likelihood. The process of the analysis was conducted by the VGLM function in R (Yee, 2010).

Methodological framework. As it might be challenging, if not possible, to highlight factors causing various impaired driving, various explanatory variables, instead, were considered and incorporated to clarify their associations with the multinomial response.
As the core concept of inclusion of various variables in this study is based on the definition of trait and attitude, those terms should be elaborated. Although personality trait is defined as a tendency to show consistent patterns of thought, behavior, or feelings (McCrae and Costa, 1995), attitudes could be defined as an evaluative condition, concerning a specific situation (Eagly and Chaiken, 1993). So, while the demographic characteristics might define the traits of an individual, attitudes are varied based on various characteristics, such as environments.

Various types of vehicles and environmental characteristics were included in the model. While demographic characteristics might define the traits of an individual; the attitudes could differ based on qualities such as variable environments. The previous studies have acknowledged this point. For instance, the importance of individual attitudes and personality traits in attributing varying importance to environmental conditions, safety, comfort, convenience, and flexibility was shown (Johansson et al., 2006). Also, in another study, it has been found that consumers' travel attitude, personality, lifestyle, and mobility are factors impacting the vehicle type choice (Chooa and Mokhtarian, 2004).

Therefore, it seems reasonable to include driver attitudes and personalities through their vehicle choice. Also, inclusion of various environmental factors are important as they trigger the way drivers operate on the roadway. In summary, while traits can be linked to driver's demographic, attitude could be associated with various environmental, roadway, or other characteristics of roadways, see Fig. 1.

Regarding the inclusion of drivers' actions in the analysis, a few points should be highlighted. First, a drivers' impairments could affect drivers' actions, so drivers' actions were included to highlight the association between driver's actions and impairments. Second, different drivers' actions result from a number of unseen choice-making behaviors before involvement in crashes, so it is reasonable to include them as independent variables.

For instance, consider the distracted drivers' response, when actions such as driving too fast for conditions might result from other confounders or unseen factors. Thus, it is important to account for the association of those factors and drivers' impairments. This point is evident in the field of health care where, while it is satisfying to elucidate components of the cause of illnesses, the more important emphasis is on the association of other factors surrounding the illness. Interested readers are referred to (Altman and Krzywinski, 2015), for more information regarding causation vs. association.

Data. The data used in this study was obtained directly from the Wyoming Department of Transportation (WYDOT), which covers years 2015-2019. There was a column highlighting the condition of drivers at the time of crashes including DUI, distraction, fatigue, and emotional conditions, vs. normal conditions as a reference category. Here, the DUI includes both drugs and alcohol. Response and all explanatory variables, which were found to be significant, are included in Table 1.

Primary there were more available predictors but not all those variables were found to be important in studying the associations with impaired driving. That is true, for instance, for roadway geometric characteristics such as vertical and horizontal curves.

Figure 2 is presented to highlight the area and topography of the case study. As can be seen from Fig. 2, the state of Wyoming is located in the western part of the US, with a mountainous topography. 


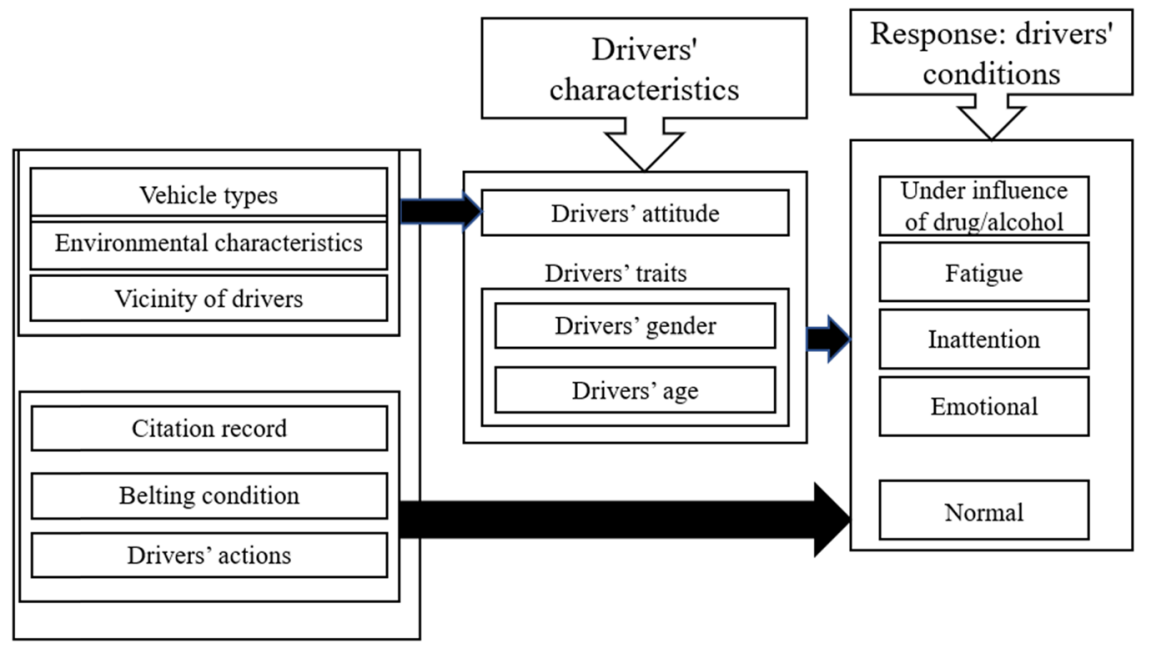

Fig. 1 Methodological framework for highlighting the associations, various factors are expected to be associated with impaired driving. However, many of those are not recorded at the time of crashes. To account for those factors, we considered, for instance, vehicle types which account for drivers attitudes, or environmental factors which highlight specific groups of drivers that are more likely to drive on those conditions.

\section{Table 1 descriptive statistics of important predictors.}

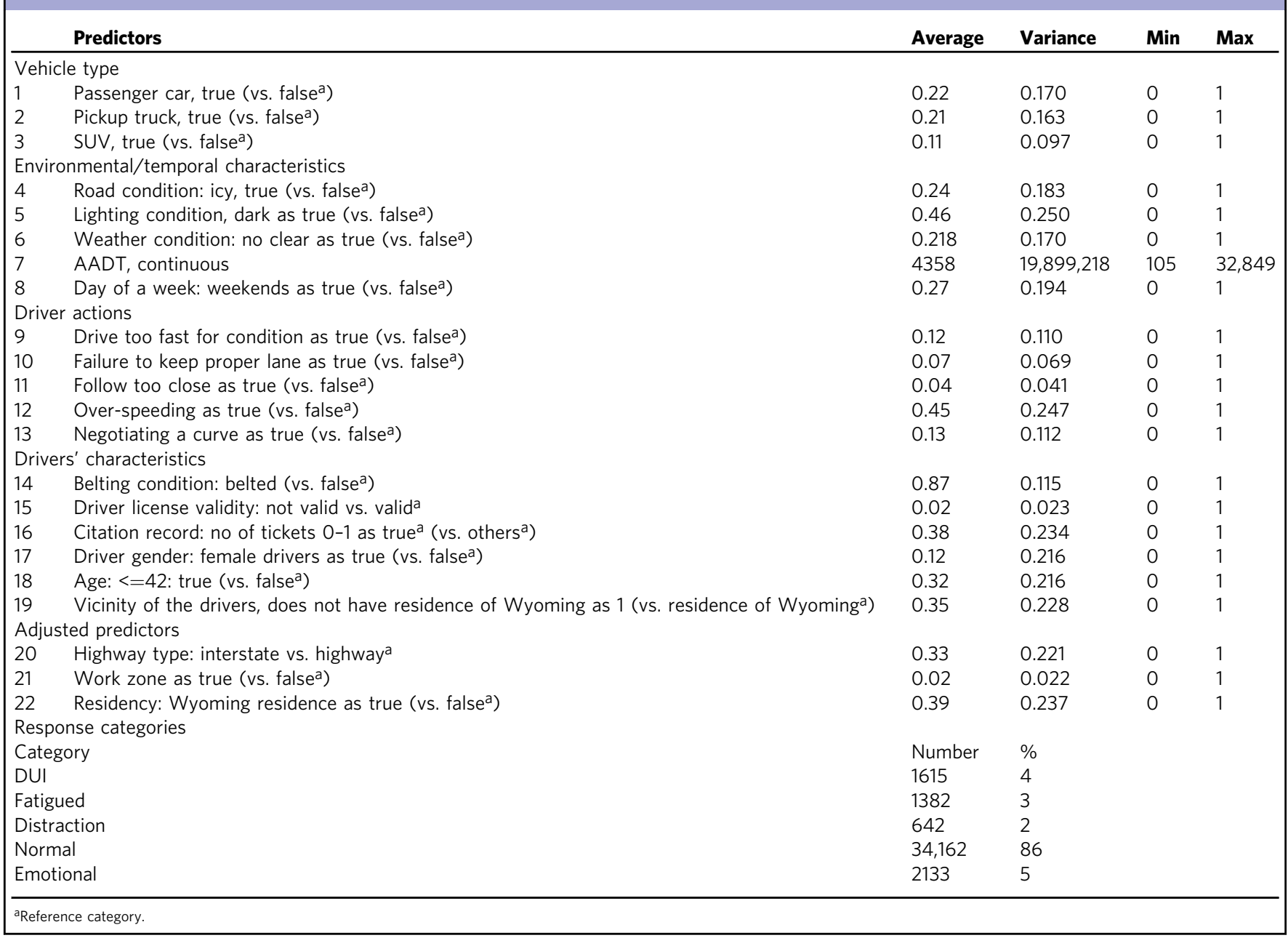

It should be highlighted that the combinations of all impaired driving account for almost half of the fatalities in the state during the study period, while those impaired driving account for $<10 \%$ of all crashes.

\section{Results}

The included factors highlight their associations with various impaired driving. Besides association consideration, it should be reiterated that crashes are expected to occur in a "noisy" 


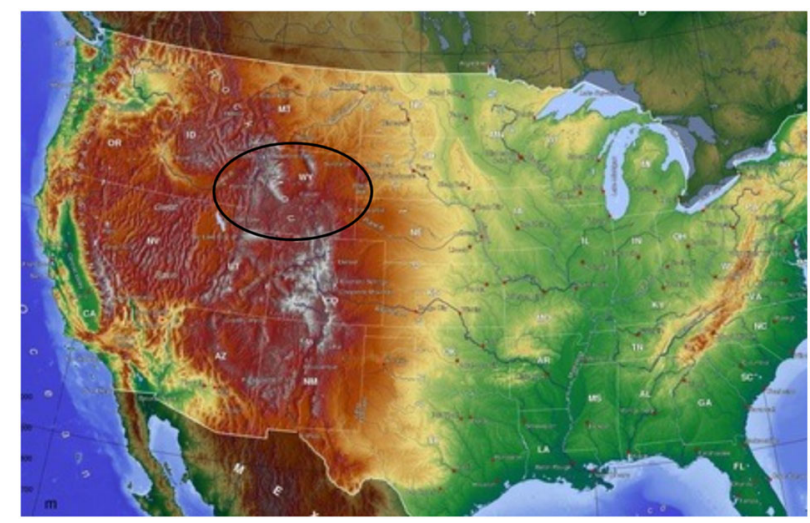

Fig. 2 Topography of Wyoming, in relation to other states, the darker areas highlight the mountainous topography.

environment with many unseen factors, and there are confounding factors that might be associated with the observed factors. Therefore, it is reasonable to include the related observed covariates to account for associations of the unseen factors. The description of this section will be presented based on the predictor's categories being considered.

Vehicle type. The vehicle types were included to account for drivers' attributes. Passenger cars, pickup, and SUVs account for 22,20 , and $11 \%$ of all vehicle types. The results highlighted that SUV drivers might be the safest drivers in various impaired driving conditions as they are negatively associated with all impaired driving types. Also, while pickup drivers are less likely to be impaired, drivers of passenger cars are positively associated with all impaired driving, except for distractions.

Environmental/temporal characteristics. Previous studies considered suboptimal weather conditions on the risk-taking behaviors of drivers. For instance, the impact of hazy conditions was investigated on risk-taking behaviors (Jiang and Rau, 2018). The questionnaire results indicated that there is an increase of aggressive driving violations, errors, and risky driving behaviors in hazy conditions. Additionally, studies show that in foggy conditions, drivers think that they are moving more slowly than they really are and consequently increase their speed (Snowden et al., 1998).

If the foggy or hazy conditions are considered as not-clear weather, our results are against the previous study as it was found that the adverse weather condition is negatively associated with all impaired driving, except for emotional drivers. It should be noted that all types of non-clear weather conditions were included as a variable of interest.

A similar explanation applies for night conditions, except that night conditions are positively associated with DUI and the emotional conditions of drivers. The positive association between DUI and night time is due to a possible reason that DUI drivers are likelier to drive at night due to a higher likelihood of drinking at a later time or prevention of getting captured by the cops.

Similarly, suboptimal road condition is negatively associated with all impaired driving; highlighting that impaired driver are likelier not to drive in those road conditions. Thus, the reason for a lower risk for suboptimal circumstances could be linked to the fact that those drivers compensate for their impaired conditions by driving in optimal conditions.

However, while dark condition is negatively associated with distracted drivers, it is positively correlated with DUI and emotional drivers. The results are partly in line with the previous study, which used completed diary entries from 15 participants and found that factors including the number of passengers, speed limit, road type, traffic conditions, weather conditions, and trip purpose are factors influencing the drivers' distraction (Parnell et al., 2020).

Work zone crashes were also negatively associated with DUI and fatigued drivers, while coefficients related to other categories were removed due to lack of significance. Also, drivers are likelier to be under the influence and drive on the interstate vs. the highway system. Owing to the significant effect of that parameter for only DUI drivers, we exclude the impacts on other response categories.

To account for exposure and understand the impact of traffic, average annual daily traffic (AADT) was considered for checking its association with impaired drivers. It was found higher traffic is negatively associated with almost all impaired driving due to the possible fact the drivers might find it riskier to drive in higher traffic due to the presence of highway patrol and its associated costs or possible involvement in crashes. The findings are against the previous study highlighting the insignificant impact of AADT on the impaired drivers vs. sober drivers (Høye, 2020).

Furthermore, although impaired driving due to DUI is positively associated with weekends, drivers are negatively associated with all other impaired driving. Highlighting those DUI drivers are likelier to drive on weekends, while other impaired drivers are likelier to drive on weekdays.

Driver actions. What drivers' actions are likelier when drivers are impaired due to various factors? For the next category, various drivers' actions were considered to verify their association with various impaired driving. For this section's description, the terms longer-term and shorter-term behaviors related to the previous study will be used; drivers' actions have been categorized into longer-term behavior including passing, following, and turning, compared with shorter-term behaviors including lane position (Pentland and Liu, 1999).

It was discussed in the literature that marijuana impairs psychomotor skills, lane tracking, and cognitive skills (Miller et al., 2020), which is in line with the findings that DUI drivers are positively associated with failure to keep the proper lane and negotiating a curve, which are shorter-term behaviors and are related to the cognitive skills.

Also, it has been highlighted that alcohol-involved drivers' judgment of speed and distance would be impaired alongside with risk-taking behaviors (Jones, 2014), which is somehow against the findings that DUI drivers are negatively associated with driving too fast for conditions, speeding, and following too close, which are all long-term behaviors. Again, the reasons for the findings might be linked to the concept of longer-term and shorter-term behaviors.

In the considered attributes, the difference between speeding and driving too fast for conditions should be noted. Speeding is related to the time that drivers involved in crashes while violating the posted speed limit. However, speed-too-fast for condition indicates that although drivers followed posted speed limit, due to the circumstances at the time of crashes, they were supposed to have a lower speed limit, which is mainly due to slippery road conditions.

Based on the results, the negative association of speed-too-fast for condition and impaired driving could be linked to the fact that drivers might compensate for the impaired impacts by not even driving on those conditions. The same explanation goes for overspeeding and following too close. Especially the positive association between over-speeding and fatigued driving might 
be expected as fatigued drivers are unlikely to reduce their speed to compensate for the impact of fatigue, but instead would increase their speed to reach their destinations so they can take a rest.

The results are somehow in line with the previous study, highlighting that fatigued driver have shown an inability to maintain a constant speed (De Valck et al., 2003). Also, our results show that fatigue is positively associated with failure to keep proper lane, which is in line with the previous study, highlighted that fatigue increases the likelihood of lane drift (Fairclough and Graham, 1999).

The result highlighted a negative association between speed-tofast for condition and speeding, and distracted drivers. The results align with the previous study using simulation, highlighting that when a driver uses his mobile phone, he will reduce his speed (Waard et al., 2001). Also, we found that driver distraction is positively associated with failure to keep proper lane, which is in line with the past study (Drews et al., 2009).

Drivers' characteristics. Drivers' demographic characteristics, such as age and gender were included as they are expected to play an important role in various types of drivers' impairment. For instance, male drivers are expected to show a higher level of risky and aggressive driving habits (Dula and Ballard, 2003), while an increase in age, or older drivers are expected to result in a decrease in certain driving styles such as risky and aggressive driving habits (Krahé and Fenske, 2002). In addition, to have a better sense about the association of various drivers' characteristics', drivers' belting condition, driver's license status, and citation record of drivers were incorporated in the analysis.

The results highlighted that non-belted drivers, and drivers with no valid drivers' license likelier to be impaired based on all considered categories, being in line with the previous study, which highlighted that speeding, no seatbelt, no valid license are associated with impaired riding (Valen et al., 2019).

While citation record of drivers is positively associated with DUI impaired drivers, they are negatively associated with other impaired driving. The preventative effects of violation monitoring and its impact on safer driving action has been discussed in the literature (Pan et al., 2020). The association of citation with DUI is somehow in line with the past study that drivers with a history of DUI are likelier to display reckless driving behaviors, and increased rate of vehicles crashes (Van Dyke and Fillmore, 2014).

Female drivers are negatively associated with all impaired driving actions, highlighting they are less likely to be impaired. Older drivers are also negatively associated with all drivers' impaired driving, except for emotional conditions. The results are against the previous study, which found that although younger drivers are less likely to be under the influence, they are likelier to just involve in a crash (Romano et al., 2012). However, in line with the previous study, which found that younger drivers, 18-24, in general are more likely to be engaged in distracting tasks (Prat et al., 2017). Finally, Wyoming residents also are less likely to be impaired drivers based on the considered response categories of DUI.

To have a vision about the performance of the modified MNL and the original MNL, various goodness of fits measures are included at the bottom of Table 2. A significant improvement in the model fit can be observed by just adjusting three variables, which justifies modifying the standard MNL model.

Finally, to improve the interpretability of the findings and to provide a better perspective for various impaired driving, Fig. 3 is provided. Similar categories as Tables 1 and 2 are provided in the figure. As can be seen and discussed in the results section, although there are many similarities across the association between various impaired driving and variables, there are significant variations.

For instance, as can be seen from Fig. 3, while there are positive associations between lighting conditions and being emotional and DUI, there is a negative association between lighting conditions and being distracted. Also, the association between lighting conditions and being fatigued is not significant, $p$-value $=0.5$. Figure 3 especially provides a useful representation to compare the associations between various impaired driving and predictors, where dashed lines highlight negative associations, while the solid lines show the positive associations. The rounded magnitude with one digit is also provided in circle.

\section{Discussion}

In the context of traffic safety, the word of impairment has been used inconsistently and with a lack of clarity of its underlying meanings. Such definitional ambiguity impedes the necessary actions to make roadways safer.

Perception regarding associated risk is especially important as they influence the decision-making processes and how we look at things (Williams and Noyes, 2007). In addition, how various situations are labeled impact the individual's assessment of the inherent risk (Smelser and Baltes, 2001). Several issues might arise from lack of comprehension or assigning less importance to the impaired driving habits. That could be seen from both policy makers' strategies and drivers' behaviors themselves.

By assuming that the impaired driving is not necessarily linked to fatigued or inattention, the policy makers would not take extreme actions like DUI, and also the drivers might assume it is ok to drive with those impaired driving habits. That is especially important as it has been noted that the perception of risk impacts the individual's decision and consequently behaviors (Siegrist et al., 2005), and those behaviors impact the safety of roadways.

For instance, Currently there is no regulation regarding fatigued driving of non-commercial vehicles, and for commercial vehicles there are only regulations for working hours of operating a commercial motor vehicle (Short, 2018), where commercial motor vehicle (CMV) applies for only school bus and other CMVs. That process limit number of driving and working hours per week. Also, it ensures drivers maintained a natural sleep/wake cycle. However, the process for non-commercial motor vehicle is only for cases of observing non-CMV for instance, changes lane abruptly or involved in crashes. As a result, they would be ticketed as improper lane change violation, for instance, which is considerably associated with lower penalty, compared with DUI. By implementation of the similar regulation for non-CMV and increasing the penalty to be in line with the DUI, it is expected that the drivers change their approaches by taking fatigued driving more seriously.

On the other hand, substantial body of research creates this misconception that although, for instance, inattention or fatigued are risky, they are not as hazardous as DUI so it should not be considered as a violation. The same misconception is shared by drivers as well so they might pay less attention to the hazard associated with impaired driving, other than DUI.

It has been noted that training and warning of the individuals are some of the steps for hazard management (Lehto, 2006). The warning or traffic enforcement could act to caution drivers regarding the presence of hazard. That consequently could prompt suitable behaviors and discourage risky actions (Hellier et al., 2000). It should be noted, while implementing the hazard management, all impaired driving should be taken into consideration.

Sobriety checkpoints for DUIs, fatigued drivers, and enforcement for significant distractions in the vehicles are suggested. 


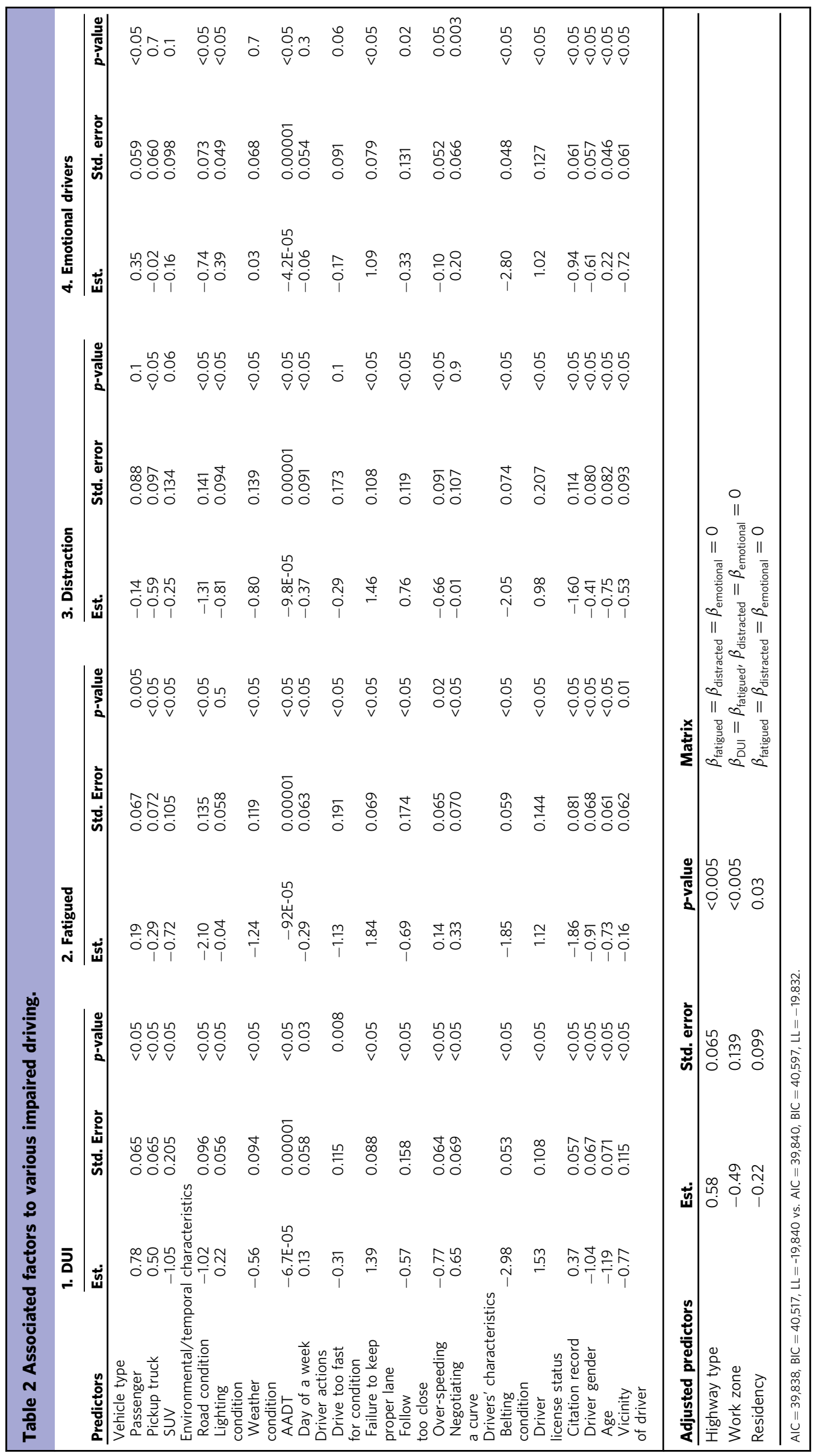




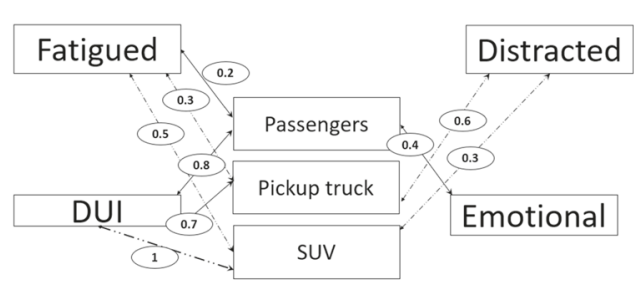

Vehicle types
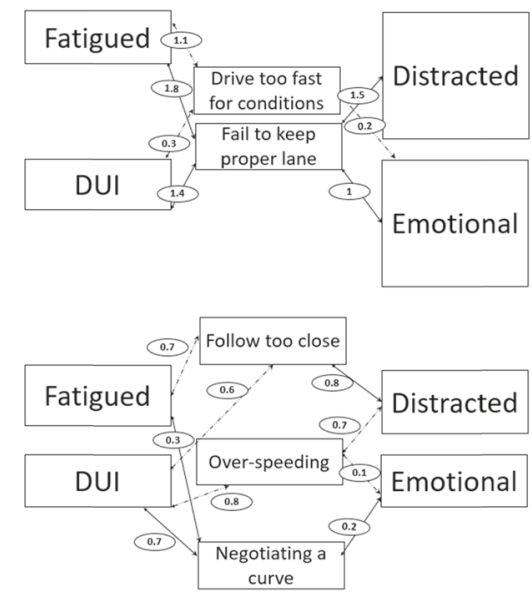

Driver actions

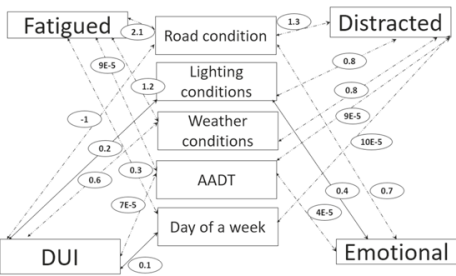

Environmental/temporal characteristics

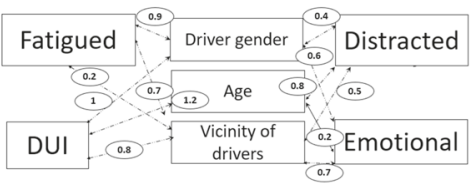

Drivers' characteristics

Fig. 3 Associations between various variables and impaired driving, dashed lines highlight negative associations, while the solid lines show the positive associations, absolute magnitude in circle, here to provide a better perspective we highlight and depict the associations in figures for a better comparison.

High visibility of highway patrol, especially during ideal weather and roadway conditions, is recommended. Failure to keep proper lane should be highlighted as a good indicator for various impaired drivers. So, more vigilance by identifying the condition of drivers committing that violation could help to stop drivers before involvement in crashes. In case of observing drivers with lack of drivers' license validity or belting condition, drivers' conditions need to be checked as based on our results, those drivers are more likely to be impaired, and thus stricter measures should be made if drivers are impaired.

The identified results could improve the understanding of the factors related to various impaired driving. The better understanding consequently would provide better educational and awareness programs to target the groups in more efficient ways. More studies are needed to incorporate all types of impaired drivers in their analysis.

The limitation of this study could be summarized as follows: the study findings, especially non-DUI impairments, was not investigated in detail by means of survey questionnaire or driving simulation datasets. Those considerations could provide a richer understanding regarding the believes and inputs of drivers while being fatigues, distracted or being under emotional conditions.

However, based on the literature review and the study findings, this study was able to provide evidence that other impaired driving behaviors are needed to be included, not only for gaining a better understanding regarding impaired driving behaviors but changing believes and perceptions regarding the impaired driving, which does not solely include DUI. As a result, policies could be adjusted to amend drivers' behaviors and actions to have safer roadways.

Although the plausibility of autonomous driving was considered in the past study (Lutin, 2018), the impaired drivers was defined only in terms of impaired with alcohol. However, based on the discussion it is expected that autonomous vehicles help not only alcohol-impaired drivers but also all types of impaired driving. However, it is especially important to gain a better perspective regarding the behavioral process of all impaired drivers' actions, before measuring the associated benefits of autonomous driving.

Media campaigns has been used as a tool for enhancing public health. Those impacts are linked on focusing on new behaviors adoption compared with cessation of problematic behaviors (Noar, 2006). For instance, the effectiveness of highly mass media campaign was investigated for promoting public health for alcohol-impaired driving crashes (Yadav and Kobayashi, 2015). However, the process is recommended to be implemented on all impaired driving, and it is expected the process to be effective in reduction of high number of injury crashes due to all types of impaired driving behaviors.

\section{Conclusion}

Overall, the results bring to light few important points that have been generally unaddressed: (i) usually impaired drivers compensate for the impaired condition by not driving in less-thanoptimal conditions, (ii) generally, there are more differences between DUI and other impaired drivers as different processes govern them, (iii) driver's license validity, belting condition and failure to keep proper lane are some of the variables that are found to be important with similar signs across all impaired driving, which could be used as indicators for impaired drivers,(iv) DUI drivers are positively associated with shorter-term behaviors such as failure to keep proper lane and negotiating curve while they are negatively associated with drive-too-fast for conditions, over-speed and follow too close. The impacts are expected to be due to performance of DUI drivers, making it more challenging to keep up with shorter-term behaviors. 
In summary, it is strongly recommended for future studies to incorporate other impaired driving, including distracted, emotional and fatigued drivers, so appropriate actions would be made not only for DUI drivers but all other impaired drivers. That is especially important due to inconsistent definition of the impaired driving in the literature.

\section{Data availability}

Data can be provided upon request.

Received: 1 September 2021; Accepted: 10 January 2022;

Published online: 19 January 2022

\section{References}

Abdel-Aty MA, Abdelwahab HT (2000) Exploring the relationship between alcohol and the driver characteristics in motor vehicle accidents. Accid Anal Prev 32(4):473-482

Abdu R, Shinar D, Meiran N (2012) Situational (state) anger and driving. Transp Res F: Traffic Psychol Behav 15(5):575-580

Altman N, Krzywinski M (2015) Association, correlation and causation. Nat Methods. 12(10):899-900

Arnold MB (1968) The nature of emotion: selected readings. Penguin (NonClassics)

Aschkenasy MT, Drescher MJ, Ratzan RM (2006) Physician reporting of medically impaired drivers. J Emerg Med 30(1):29-39

Berger JT, Rosner F, Kark P, Bennett AJ (2000) Reporting by physicians of impaired drivers and potentially impaired drivers. J Gen Intern Med 15(9):667-672

Blincoe LJ, Miller TR, Zaloshnja E, Lawrence BA (2015) The economic and societal impact of motor vehicle crashes, 2010.(Revised)(Report No. DOT HS 812 013). National Highway Traffic Safety Administration, Washington, DC

Choo S, Mokhtarian PL (2004) What type of vehicle do people drive? The role of attitude and lifestyle in influencing vehicle type choice. Transp Res A: Policy Pract 38(3):201-222

Cummings P, Koepsell TD, Moffat JM, Rivara FP (2001) Drowsiness, countermeasures to drowsiness, and the risk of a motor vehicle crash. Inj Prev 7(3):194-199

De Valck E, De Groot E, Cluydts R (2003) Effects of slow-release caffeine and a nap on driving simulator performance after partial sleep deprivation. Percept Mot Skills 96(1):67-78

Deffenbacher JL, Deffenbacher DM, Lynch RS, Richards TL (2003) Anger, aggression, and risky behavior: a comparison of high and low anger drivers. Behav Res Ther 41(6):701-718

Drews FA, Yazdani H, Godfrey CN, Cooper JM, Strayer DL (2009) Text messaging during simulated driving. Hum Factors 51(5):762-770

Dula CS, Ballard ME (2003) Development and evaluation of a measure of dangerous, aggressive, negative emotional, and risky driving 1. J Appl Soc Psychol 33(2):263-282

Eagly AH, Chaiken S (1993) The psychology of attitudes. Harcourt brace Jovanovich college publishers

Eboli L, Mazzulla G, Pungillo G (2017) The influence of physical and emotional factors on driving style of car drivers: a survey design. Travel Behav Soc 7:43-51

Elvik R (2013) Risk of road accident associated with the use of drugs: a systematic review and meta-analysis of evidence from epidemiological studies. Accid Anal Prev 60:254-267

Fairclough SH, Graham R (1999) Impairment of driving performance caused by sleep deprivation or alcohol: a comparative study. Hum Factors 41(1):118-128

Fernandes R, Hatfield J, Job RS (2010) A systematic investigation of the differential predictors for speeding, drink-driving, driving while fatigued, and not wearing a seat belt, among young drivers. Transp Res F: Traffic Psychol Behav 13(3):179-196

Green PJ (1984) Iteratively reweighted least squares for maximum likelihood estimation, and some robust and resistant alternatives. J R Stat Soc: Ser B (Methodol) 46(2):149-170

Hellier E, Wright DB, Edworthy J, Newstead S (2000) On the stability of the arousal strength of warning signal words. Appl Cogn Psychol 14(6):577-592

Høye A (2020) Speeding and impaired driving in fatal crashes-Results from indepth investigations. Traffic Inj Prev 21(7):425-430

Hu T, Xie X, Li J (2013) Negative or positive? The effect of emotion and mood on risky driving. Transp Res F: Traffic Psychol Behav 16:29-40

Jiang C, Rau PP (2018) Rule obedience as a mediator between normlessness and risky driving in hazy conditions. Transp Res F: Traffic Psychol Behav 59:188-194
Johansson MV, Heldt T, Johansson P (2006) The effects of attitudes and personality traits on mode choice. Transp Res A: Policy Pract 40(6):507-525

Jones AW (2014) Effects of alcohol on fitness to drive. In: Handbook of forensic medicine. 1057-1073

Krahé B, Fenske I (2002) Predicting aggressive driving behavior: The role of macho personality, age, and power of car. Aggress Behav 28(1):21-29

Lehto MR (2006) Human factors models. In: Handbook of warnings, 63-87

Lerner BH (2011) Drunk driving, distracted driving, moralism, and public health. N Engl J Med 365(10):879-881

Lowrie J, Brownlow H (2020) The impact of sleep deprivation and alcohol on driving: a comparative study. BMC Public Health 20(1):1-9

Lutin JM (2018) Not if, but when: autonomous driving and the future of transit. J Public Transp 21(1):10

Lyznicki JM, Doege TC, Davis RM, Williams MA (1998) Sleepiness, driving, and motor vehicle crashes. JAMA 279(23):1908-1913

McCrae RR, Costa Jr PT (1995) Trait explanations in personality psychology. Eur J Person 9(4):231-252

McEvoy SP, Stevenson MR, Woodward M (2006) The impact of driver distraction on road safety: results from a representative survey in two Australian states. Inj Prev 12(4):242-247

McFadden D (1973) Conditional logit analysis of qualitative choice behavior

Miller RE, Brown TL, Lee S, Tibrewal I, Gaffney GG, Milavetz G, Hartman RL, Gorelick DA, Compton R, Huestis MA (2020) Impact of cannabis and low alcohol concentration on divided attention tasks during driving. Traffic Inj Prev 21(sup1):S123-S129

National Highway Traffic Safety Administration (2017) Traffic Safety Facts, Alcohol-impaired Driving. National Highway Traffic Safety Administration

Nelson SE, Shoov E, LaBrie RA, Shaffer HJ (2019) Externalizing and self-medicating: Heterogeneity among repeat DUI offenders. Drug Alcohol Depen 194:88-96

Noar SM (2006) A 10-year retrospective of research in health mass media campaigns: where do we go from here? J Health Commun 11(1):21-42

Oviedo-Trespalacios O, Haque MM, King M, Washington S (2017) Self-regulation of driving speed among distracted drivers: An application of driver behavioral adaptation theory. Traffic Inj Prev 18(6):599-605

Pan F, Yang Y, Zhang L, Ma C, Yang J, Zhang X (2020) Analysis of the impact of traffic violation monitoring on the vehicle speeds of urban main road: taking China as an example. J Adv Transport 2020: 6304651

Parnell KJ, Rand J, Plant KL (2020) A diary study of distracted driving behaviours. Transp Res F: Traffic Psychol Behav 74:1-14

Pentland A, Liu A (1999) Modeling and prediction of human behavior. Neural Comput 11(1):229-242

Prat F, Gras ME, Planes M, Font-Mayolas S, Sullman M (2017) Driving distractions: an insight gained from roadside interviews on their prevalence and factors associated with driver distraction. Transp Res F: Traffic Psychol Behav 45:194-207

Regan MA, Mitsopoulos E (2001) Understanding passenger influences on driver behaviour: implications for road safety and recommendations for countermeasure development

Romano EO, Peck RC, Voas RB (2012) "Traffic environment and demographic factors affecting impaired driving and crashes". J Safe Res 43(1):75-82

Safety H (2003) Research continues on a variety of factors that contribute to motor vehicle crashes

Sagberg F (2006) Driver health and crash involvement: a case-control study. Accid Anal Prev 38(1):28-34

Sheykhfard A, Haghighi F (2020) Driver distraction by digital billboards? Structural equation modeling based on naturalistic driving study data: a case study of Iran. J Safe Res 72:1-8

Short J (2018) Technical memorandum: hours-of-service flexibility

Siegrist M, Gutscher H, Earle TC (2005) Perception of risk: the influence of general trust, and general confidence. J Risk Res 8(2):145-156

Smelser NJ, Baltes PB (2001) International encyclopedia of the social \& behavioral sciences. Elsevier, Amsterdam

Snowden RJ, Stimpson N, Ruddle RA (1998) Speed perception fogs up as visibility drops. Nature 392:6675. 450

Stutts JC, Wilkins JW, Osberg JS, Vaughn BV (2003) Driver risk factors for sleeprelated crashes. Accid Anal Prev 35(3):321-331

Subramanian R (2005) Motor vehicle traffic crashes as a leading cause of death in the United States, 2001. Young 1:3

The National Sleep Foundation (2021) Drowsy driving prevention. The National Sleep Foundation

Turnbull PR, Khanal S, Dakin SC (2021) The effect of cellphone position on driving and gaze behaviour. Sci Rep 11(1):1-10

Valen A, Bogstrand ST, Vindenes V, Frost J, Larsson M, Holtan A, Gjerde H (2019) Driver-related risk factors of fatal road traffic crashes associated with alcohol or drug impairment. Accid Anal Prev 131:191-199

Van Dyke N, Fillmore MT (2014) Alcohol effects on simulated driving performance and self-perceptions of impairment in DUI offenders. Exp Clin Psychopharmacol 22(6):484 
Waard DD, Brookhuis KA, Hernandez-Gress N (2001) The feasibility of detecting phone-use related driver distraction. Int J Veh Des 26(1):85-95

Williams DJ, Noyes JM (2007) How does our perception of risk influence decisionmaking? Implications for the design of risk information. Theor Issues Ergon Sci 8(1):1-35

World Health Organization and the association for safe international road travel (2007) Faces behind the figures: voices of road traffic victims and their families. World Health Organization and the association for safe international road travel

Yadav R, Kobayashi M (2015) A systematic review: effectiveness of mass media campaigns for reducing alcohol-impaired driving and alcohol-related crashes. BMC Public Health 15(1):1-17

Yee TW (2010) The VGAM package for categorical data analysis. J Stat Softw $32(10): 1-34$

Zhang X, Zhao X, Du H, Rong J (2014) A study on the effects of fatigue driving and drunk driving on drivers' physical characteristics. Traffic Inj Prev 15(8):801-808

Zhao S, Khattak AJ (2018) Injury severity in crashes reported in proximity of rail crossings: the role of driver inattention. J Transp Safe Secur 10(6):507-524

\section{Competing interests}

The authors declare no competing interests.

\section{Ethical approval}

Not applicable.

\section{Informed consent}

Not applicable.

\section{Additional information}

Correspondence and requests for materials should be addressed to Mahdi Rezapour.

Reprints and permission information is available at http://www.nature.com/reprints

Publisher's note Springer Nature remains neutral with regard to jurisdictional claims in published maps and institutional affiliations.

(c) (i) Open Access This article is licensed under a Creative Commons Attribution 4.0 International License, which permits use, sharing, adaptation, distribution and reproduction in any medium or format, as long as you give appropriate credit to the original author(s) and the source, provide a link to the Creative Commons license, and indicate if changes were made. The images or other third party material in this article are included in the article's Creative Commons license, unless indicated otherwise in a credit line to the material. If material is not included in the article's Creative Commons license and your intended use is not permitted by statutory regulation or exceeds the permitted use, you will need to obtain permission directly from the copyright holder. To view a copy of this license, visit http://creativecommons.org/ licenses/by/4.0/.

(C) The Author(s) 2022 